International Journal of Pure and Applied Mathematics

Volume 82 No. 5 2013, 821-824

ISSN: 1311-8080 (printed version); ISSN: 1314-3395 (on-line version)

url: http://www.ijpam.eu

doi: http://dx.doi.org/10.12732/ijpam.v82i5.10

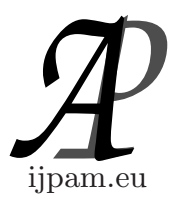

\title{
YET ANOTHER $X$-RANK \\ CHARACTERIZATION OF RATIONAL NORMAL CURVES
}

\author{
E. Ballico \\ Department of Mathematics \\ University of Trento \\ 38123 Povo (Trento) - Via Sommarive, 14, ITALY
}

\begin{abstract}
Fix positive integers $s, k_{i}, 1 \leq i \leq s$, such that $k_{1} \geq 2$ and $2 k<n$, where $k:=k_{1}+\cdots+k_{s}$. Let $X \subset \mathbb{P}^{n}$ be an integral and non-degenerate curve. For any $P \in \mathbb{P}^{n}$ the $X$-rank $r_{X}(P)$ of $P$ is the minimal cardinality of a set $S \subset X$ such that $P \in\langle S\rangle$. We prove that $X$ is not a rational normal curve if and only if the following condition holds: fix $s$ general points $P_{1}, \ldots, P_{s} \in X_{\text {reg }}$ and set $Z:=\sum_{i=1}^{s} k_{i} P_{i}$; then there is some $P \in\langle Z\rangle$ such that $P \notin\left\langle Z^{\prime}\right\rangle$ for any $Z^{\prime} \subsetneq Z$ and $r_{X}(P) \leq n+1-k$.

Moreover, if $X$ is not a rational normal curve and we fix a finite set $E \subset X$, then we may find a set $S \subset X \backslash E$ with $\sharp(S) \leq n+1-k$ and $P \in\langle S\rangle$.
\end{abstract}

AMS Subject Classification: $14 \mathrm{H} 50,14 \mathrm{~N} 05$

Key Words: $X$-rank, rational normal curve

\section{Introduction}

Let $X \subseteq \mathbb{P}^{n}$ be an integral and non-degenerate variety defined over an algebraically closed field $\mathbb{K}$. For any $P \in \mathbb{P}^{n}$ the $X$-rank $r_{X}(P)$ of $P$ is the minimal cardinality of a finite set $S \subset X$ such that $P \in\langle S\rangle$, where \langle\rangle denote the linear span. In characteristic zero we have $r_{X}(P) \leq n+1-\operatorname{dim}(X)$ for any $X$ and any $P$ (see [5], Proposition 4.1). In positive characteristic this is true, except

Received: December 14, 2012

(c) 2013 Academic Publications, Ltd. url: www.acadpubl.eu 
for at most one $P$ (see [1]). In this short note we prove the following result.

Theorem 1. Fix positive integers $s, k_{i}, 1 \leq i \leq s$, such that $k_{1} \geq 2$ and $2 k<n$, where $k:=k_{1}+\cdots+k_{s}$. Let $X \subset \mathbb{P}^{n}$ be an integral and non-degenerate curve. $X$ is not a rational normal curve if and only if the following condition \$ holds:

Condition : Fix s general points $P_{1}, \ldots, P_{s} \in X_{\text {reg }}$ and set $Z:=\sum_{i=1}^{s} k_{i} P_{i}$. Then there is some $P \in\langle Z\rangle$ such that $P \notin\left\langle Z^{\prime}\right\rangle$ for any $Z^{\prime} \subsetneq Z$ and $r_{X}(P) \leq$ $n+1-k$.

Moreover, if $X$ is not a rational normal curve and we fix a finite set $E \subset X$, then we may find a set $S \subset X \backslash E$ with $\sharp(S) \leq n+1-k$ and $P \in\langle S\rangle$.

In the case $k_{i}=1$ for all $i$ we prove the following result.

Theorem 2. Fix integers $k \geq 2$ and $n>2 k$. Let $X \subset \mathbb{P}^{n}$ be an integral and non-degenerate curve. Fix a general $Z \subset X$ such that $\sharp(Z)=s$. $X$ is not a rational normal curve if and only if there is some $P \in\langle Z\rangle$ such that $P \notin\left\langle Z^{\prime}\right\rangle$ for any $Z^{\prime} \subsetneq Z$ and $W \supseteq Z$ for every set $W \subset X$ such that $\sharp(W) \leq n+1-k$, $W \cap Z=\emptyset$ and $P \in\langle W\rangle$. Moreover, if $X$ is not a rational normal curve for any fixed finite set $E \subset X$ we may find $W$ as above with $W \cap E=\emptyset$.

Instead of $W \cap Z=\emptyset$ we may assume $W \nsupseteq Z$.

Question 1. For general $Z$ is it possible to take as $P$ a general element of $\langle Z\rangle$ ? Or, even, every $P \in\langle Z\rangle$ such that $P \notin\left\langle Z^{\prime}\right\rangle$ for any $Z^{\prime} \subsetneq Z$ ?

\section{The Proofs}

Lemma 1. Let $C \subset \mathbb{P}^{r}$ be an integral and non-degenerate curve. Fix a general $P \in C$ and a finite set $E \subset C$ with $P \notin E$. There is a finite set $S \subset C \backslash E$ such that $P \in\langle S\rangle, P \notin S$ and $\sharp(S) \leq r$ if and only if $C$ is not a rational normal curve.

Proof. If $C$ is a rational normal, then no such set exists, because any $r+1$ points of $C$ are linearly independent. Now assume that $C$ is a rational normal curve. Take a general hyperplane $H \subset \mathbb{P}^{r}$ containing $P$. For general $H$ we have $H \cap E=\emptyset$. Since $P$ is general in $C, H$ may be seen as a general hyperplane. Hence $C \cap H$ is a general hyperplane section of $C$. Hence $C \cap H$ is formed by $\operatorname{deg}(C)>n$ distinct points and $C \cap H$ spans $H$. If $C$ is not very strange in the sense of [6], then we may take as $S$ any $r$ points of $C \cap H \backslash\{P\}$. If $C$ is very strange, then we need to check that not all $r$-ples of point of $C \cap H$ 
spanning $H$ contains $P$. Fix $P_{1}, P_{2} \in C \cap H \backslash\{P\}$ such that $P_{1} \neq P_{2}$. If $r=2$ we take $S=\left\{P_{1}, P_{2}\right\}$. Assume $r>2$. For every integer $t \in\{2, \ldots, r-2\}$ all $t$-dimensional linear subspaces of $H$ spanned by points of $C \cap H$ contain the same number of points of $C \cap H$. Hence there is $P_{3} \in C \cap H \backslash\left(\left\langle P_{1}, P_{2}\right\rangle \cup\{P\}\right)$. And so on if $r>3$.

Proof of Theorems 1 and 2. In characteristic zero the "if part of Theorem 1 is the easy part of theorem of Sylvester (see [4], [3], [5]). In arbitrary characteristic it is just [2], Lemma 1 , and the observation that if $X$ is a rational normal curve, then every zero-dimensional scheme $A \subset X$ with $\operatorname{deg}(A) \leq n+1$ is linearly independent. Hence it is sufficient to prove the "only if " part. In the set-up of Theorem 2 take $s:=k$ and write $Z=\left\{P_{1}, \ldots, P_{s}\right\}$. Fix a finite set $E \subset X$. Let $\ell: \mathbb{P}^{n} \backslash\left\{P_{s}\right\} \rightarrow \mathbb{P}^{n-1}$ denote the linear projection from $P_{s}$. Since $P_{s}$ is general in $X$, a dimensional count gives that for a general $Q \in X$ the line $\left.\left\{Q, P_{s}\right\}\right\rangle$ meets $X$ only at $Q$ and $P_{s}$ and that a general tangent line of $X$ does not contain $P_{s}$. Hence $\ell \mid X \backslash\left\{P_{s}\right\}$ is birational onto its image. Let $C \subset \mathbb{P}^{n-1}$ denote the closure of $\ell\left(X \backslash\left\{P_{s}\right\}\right)$ in $\mathbb{P}^{n-1}$. Since $\operatorname{deg}(C)=\operatorname{deg}(X)-1, X$ is a rational normal curve if and only if $C$ is a rational normal curve. Assume that $C$ is not a rational normal curve. Set $Q_{i}:=\ell\left(P_{i}\right), 1 \leq i \leq s-1$. Let $Q_{s} \in C$ be the only point corresponding to the tangent line of $X$ at $P_{s}$. Set $E^{\prime}:=\ell\left(E \backslash\left\{P_{s}\right\}\right) \cup\left\{Q_{1}, \ldots, Q_{s}\right\}$. Set $B:=\sum_{i=1}^{s-1} k_{i} Q_{i}+\left(k_{s}-1\right) Q_{s}$ with the convention that $0 Q_{s}$ is the zero divisor $C$. Since $P_{1}, \ldots, P_{s}$ are general in $X$, $Q_{1}, \ldots, Q_{s}$ are general in $C$. We use induction on $k$. First assume $k=2$. In this case $B=Q_{1}$. Lemma 1 with the set $E^{\prime}$ gives the existence of a set $S^{\prime} \subset C \backslash\left(E_{1} \cup Z_{r e d}\right)$ such that $\sharp\left(S^{\prime}\right) \leq n+1-k$ and $Q_{1} \in\left\langle S^{\prime}\right\rangle$. Since $Q_{s} \notin S$, there is a unique set $S \subset X$ such that $\ell(S)=S^{\prime}$. By construction we have $S \cap E=\emptyset$. Since $Q_{1} \in\left\langle S^{\prime}\right\rangle$ and $\ell$ is the linear projection from $P_{1}$, we have $\langle Z\rangle \cap\langle S\rangle$. Since $P_{s} \notin S$ and $P_{1} \notin S$, the set $\langle Z\rangle \cap\langle S\rangle$ is a unique point, $P$, and $P \notin\left\langle Z^{\prime}\right\rangle$ for any $Z^{\prime} \subsetneq Z$. Now assume $k>2$. We apply the inductive assumption the integer $k-1$. Take $S^{\prime} \subset C \backslash S^{\prime}$ such that $\sharp\left(S^{\prime}\right) \leq n-1-(k-1)=n-k$ and $\left\langle S^{\prime}\right\rangle \cap\langle B\rangle$ contains a point $P^{\prime}$ such that $P^{\prime} \notin\left\langle B^{\prime}\right\rangle$ for any $B^{\prime} \subsetneq B$. Then as in the case $k=2$ we may take as $S$ the only subset of $X$ with $\ell(S)=S^{\prime}$.

\section{Acknowledgements}

The author was partially supported by MIUR and GNSAGA of INdAM (Italy). 
E. Ballico

\section{References}

[1] E. Ballico, An upper bound for the $X$-ranks of points of $\mathbb{P}^{n}$ in positive characteristic, Albanian J. Math., 5, No. 1 (2011), 3-10.

[2] E. Ballico, A. Bernardi, Decomposition of homogeneous polynomials with low rank, Math. Z., 271 (2012), 1141-1149.

[3] A. Bernardi, A. Gimigliano, M. Idà, On the stratification of secant varieties of Veronese varieties viasymmetric rank, J. Symbolic. Comput., 46 (2011), 34-55.

[4] G. Comas, M. Seiguer, On the rank of a binary form, Found. Comp. Math., 11, No. 1 (2011), 65-78.

[5] J.M. Landsberg, Z. Teitler, On the ranks and border ranks of symmetric tensors, Found. Comput. Math., 10 (2010), 339-366, doi: 10.1007/510208009-9055-3.

[6] J. Rathmann, The uniform position principle for curves in characteristic p, Math. Ann., 276, No. 4 (1987), 565-579. 